\title{
Subjective Taste and Smell Changes in Conjunction with Anxiety and Depression Are Associated with Symptoms in Patients with Functional Constipation and Irritable Bowel Syndrome
}

\author{
Jie Liu $\mathbb{D}^{1},{ }^{1}$ Chaolan Lv, ${ }^{1}$ Dandan Wu, ${ }^{2}$ Ying Wang, ${ }^{2}$ Chenyu Sun, ${ }^{3}$ Ce Cheng, \\ and Yue Yu iD ${ }^{1,2}$ \\ ${ }^{1}$ Department of Gastroenterology, the First Affiliated Hospital of USTC, Division of Life Sciences and Medicine, University of Science \\ and Technology of China, Hefei, Anhui 230001, China \\ ${ }^{2}$ South District of Endoscopic Center, the First Affiliated Hospital of USTC, Division of Life Sciences and Medicine, University of \\ Science and Technology of China, Hefei, Anhui 230001, China \\ ${ }^{3}$ Internal Medicine, AMITA Health Saint Joseph Hospital Chicago, 2900 N. Lake Shore Drive, Chicago, 60657 Illinois, USA \\ ${ }^{4}$ The University of Arizona College of Medicine at South Campus, 2800 E Ajo Way, Tucson, 85713 AZ, USA
}

Correspondence should be addressed to Yue Yu; yuyuemd@163.com

Received 9 April 2021; Accepted 9 September 2021; Published 20 September 2021

Academic Editor: Tatsuya Toyokawa

Copyright @ 2021 Jie Liu et al. This is an open access article distributed under the Creative Commons Attribution License, which permits unrestricted use, distribution, and reproduction in any medium, provided the original work is properly cited.

Background. Patients with functional constipation (FC) and irritable bowel syndrome (IBS) often report psychological abnormalities and decreased eating enjoyment. Several patients also complain of changes in the sense of smell and taste, but these are often disregarded clinically. Aims. Therefore, there is a need to determine whether taste/smell disturbances and psychological abnormalities are present in patients with FC or IBS and whether these are related to the severity of lower gastrointestinal symptoms. Methods. A total of 337 subjects were recruited, including FC $(n=115)$, IBS $(n=126)$, and healthy controls $(n=96)$. All participants completed questionnaires evaluating taste and smell (taste and smell survey (TSS)), Lower Gastrointestinal Symptoms Rating Scale (LGSRS), Hamilton anxiety scale (HAMA), and Hamilton depression scale (HAMD). TSS recorded information on the nature of taste and smell changes (TSCs) and the impact of these changes on the quality of life. LGSRS was used to assess the severity of lower gastrointestinal symptoms; HAMA and HAMD scales were used to reflect the psychosocial state. This study protocol was registered on the Chinese Clinical Trial Registry (No. ChiCTR-2100044643). Results. Firstly, we found that taste and smell scores were higher in patients with IBS than in healthy controls. Secondly, for FC and IBS patients, LGSRS was significantly correlated with the taste score (Spearman's rho $=0.832, P<0.001$ ). LGSRS was also significantly correlated with HAMA (Spearman's rho $=0.357, P=0.017$ ) and HAMD (Spearman's rho $=0.377, P=0.012$ ). In addition, the taste score was significantly correlated with HAMD (Spearman's rho $=0.479, P=0.001$ ), while the smell score was also significantly correlated with HAMD (Spearman's rho $=0.325, P=0.031$ ). Thirdly, $60.87 \%$ and $71.43 \%$ of patients complained of taste abnormality, while $65.22 \%$ and $71.43 \%$ had smell abnormality in the FC and IBS groups, respectively. Meanwhile, $47.83 \%$ and $47.62 \%$ of patients suffered from anxiety, while $43.48 \%$ and $57.14 \%$ suffered from depression in the FC and IBS groups, respectively. Finally, we found significant differences in the taste, smell, HAMD, and LGSRS scores between the female and male IBS groups $(P<0.050)$. Conclusions. TSCs and psychological disorders are prominent in FC and IBS patients. Taste abnormalities, as well as anxiety and depression, are significantly correlated with LGSRS. Awareness of this high prevalence of taste/smell abnormalities and the psychological changes among patients with FC and IBS may help better predict and understand the severity of symptoms. 


\section{Introduction}

It is recognized that an increasing number of patients are suffering from chronic gastrointestinal diseases worldwide $[1,2]$. In a recent survey, $40.0 \%$ of patients with gastroparesis and $60.0 \%$ of patients with gastroesophageal reflux disease (GERD) scored their taste abnormalities as either mild to moderate or severe to intolerable, respectively [3]. Kabadi et al. [3] found significant abnormalities in taste and smell in patients with gastroparesis and GERD, and this change was significantly correlated with upper gastrointestinal symptoms. In patients with inflammatory bowel disease, the smell function was significantly lower than the general population, even though patients themselves were not aware of their reduced olfactory/gustatory function [4]. Therefore, it is necessary to understand the relationship between chemical sensation and digestive tract symptoms.

Disorders in smell and taste were reported to occur more frequently in the elderly, and such disorders influence nutrition, safety, quality of life, and physical and mental health [5]. Bernhardson et al. [6] reported that TSCs caused pain and reduced quality of life. Because dining is not only for nutrition intake but also has important symbolic, cultural, and religious values that may affect the psychological and social aspects of life. TSCs of patients may result in chronic unconscious weight loss and malnutrition, secondary to the change of food enjoyment, dietary preferences, and dietary intake $[7,8]$. Therefore, the severity of lower gastrointestinal symptoms and characteristics of TSCs and psychological abnormality should be assessed to better understand and support patients.

It is worth noting that depression may result or cause gustatory and olfactory dysfunction. TSCs may cause negative emotions in cancer patients, including disappointment, depression, and sadness $[9,10]$. One previous study analyzed the data obtained from a nationally representative probability sample of 3005 older American adults and reported an association between any depressive symptoms and olfactory dysfunction [11]. Moreover, a meta-analysis study evaluated the relationship between depression and TSCs, and the authors concluded that depressed individuals had more deterioration of olfaction than nondepressed controls [12]. Kohli et al. [13] included 10 studies and demonstrated that patients with depression had reduced olfactory performance compared with the healthy controls. Conversely, patients with olfactory dysfunction have symptoms of depression that worsen with the severity of smell loss. Another U.S. population-based survey detected a strong association between major depression and alterations in smell and taste [14]. On the other hand, globus sensation, a functional gastrointestinal disease, often arises concomitantly with psychological comorbidities, including major depression [15]. However, most of the previous studies on TSCs and chronic digestive diseases had ignored the effect of psychological comorbidities $[3,4]$.

In this study, taste and smell survey (TSS) was used to evaluate TSCs in patients with functional constipation (FC) and IBS, while Lower Gastrointestinal Symptoms Rating
Scale (LGSRS) was used to assess the severity of lower digestive tract symptoms. The Hamilton anxiety scale (HAMA)/Hamilton depression scale (HAMD) was then used to measure the psychological state of the patients. This study compared the TSCs, the severity of lower gastrointestinal symptoms, and the scores of patients' anxiety and depression scale with those of healthy controls. In addition, we further explored the association among TSCs, the severity of lower gastrointestinal symptoms, and the anxiety and depression scale's scores.

\section{Methods}

2.1. Patients. Patients (outpatients and inpatients) who met the Rome IV diagnostic criteria [16] for FC and IBS were diagnosed by a gastroenterologist (Y Y) experienced in the diagnosis of functional digestive disorders and were recruited into the study at the Center of Gastrointestinal Motility, the First Affiliated Hospital of University of Science and Technology of China from January 2018 to August 2020. The inclusion criteria of FC and IBS were used based on the Rome IV criteria [17-19]. Meanwhile, colonoscopy or barium enema was performed within the first four weeks, and organic intestinal lesions have been excluded.

The exclusion criteria included the following: (i) severe heart and lung diseases, diabetes, nephropathy, and other chronic diseases associated with neuropathy or gastrointestinal disorders such as ulcers, cancer, and esophageal varices; (ii) patients who were taking drugs that could affect mental status within one month such as antidepressants, corticosteroids, or sedative-hypnotics; and (iii) participants who had diseases that could affect taste and smell sensation, including patients with malignant tumors who were taking antitumor drugs, as well as allergic patients who were taking antiallergic drugs and so on.

This study protocol was registered on the Chinese Clinical Trial Registry (No. ChiCTR-2100044643), and the study was approved by the Institutional Review Board of the First Affiliated Hospital of University of Science and Technology of China (Ethics Committee of the First Affiliated Hospital of University of Science and Technology of China). In addition, all patients signed a written informed consent before being included in the study.

115 patients with FC and 126 patients with IBS were collected as research objects surveyed in the Anhui Provincial Hospital from January 2018 to August 2020. Meanwhile, 96 healthy subjects were recruited from accompanying persons of included patients after advertisement in the First Affiliated Hospital of University of Science and Technology of China and were considered the control group.

2.2. Demographics Questionnaire. The patients were subjected to a questionnaire which asked about their age, gender, and length of diagnosis with FC and IBS. Participants' weight, in kilograms, divided by their height, in meters squared, was used for calculating body mass index (BMI). Subjects also listed all current medicines, tests that had been done, past medical conditions, and past surgeries. 
2.3. Taste and Smell Survey. Taste and smell survey was compiled by Heald et al. [16] for HIV patients. There were 9 questions related to taste change and 5 questions related to smell change. TSS was widely used to assess changes in taste and smell in cancer patients [20-22]. The taste survey consisted of 9 items. Subjects were asked whether there was a change in taste self-perception (stronger or weaker) in salty, sweet, sour, and bitter tastes and the extent of this change (insignificant, mild to moderate, and severe to intolerable). 8 items scored $0-1$ points and 1 item scored $0-2$ points, with a total score of 10 points. The smell survey consisted of 5 items. Subjects were asked whether there was a change in smell self-perception, whether a particular food smells stronger or weaker and the extent of this change (insignificant, mild to moderate, and severe to intolerable). 4 items scored 0-1 points and 1 item scored 0-2 points, with a total score of 6 points. For all the questions, subjects were asked to compare their taste and smell senses at the time of data collection to the time before they were diagnosed with FC and IBS. Healthy controls were asked to compare their taste and smell senses to 10 years prior. The internal consistency reliability of previous search report was 0.89 [22].

2.4. Lower Gastrointestinal Symptoms Score. LGSRS was revised according to the gastrointestinal symptom rating scale [23]. There were 15 questions in the original score table, including five aspects of gastrointestinal symptoms: abdominal pain (including abdominal pain, nausea, and vomiting), reflux symptoms (including heartburn and acid reflux), diarrhea, symptoms of dyspepsia (including diarrhea, loose stool, fecal incontinence, and sense of urgency in defecation), symptoms of indigestion (including bellyache, abdominal distention, belching, and increased exhaust), and symptoms of constipation (including constipation, hard stool, and incomplete defecation). Each question provided four choices, and each symptom is scored separately from light to heavy, with a score of 0-3: 0 (asymptomatic), 1 (mild symptoms), 2 (moderate symptoms), and 3 (severe symptoms), and finally, each item's score was summed to obtain a total score. The higher the score, the more serious the gastrointestinal symptoms were. According to the above contents, 10 indicators, including periumbilical pain, diarrhea, constipation, loose stool, fecal incontinence, incomplete defecation, lower abdominal distention, abnormal bowel sounding, increased exhaust, and hard stool, were selected as the indicators of the lower gastrointestinal symptoms rating scale (LGSRS), with a cumulative score of LGSRS. All the scales were completed independently within 10 to 15 minutes and were guided by doctors trained in a unified way to review the symptoms within one week. Cronbach's alpha coefficients were used for measuring the internal consistency reliability and validity of LGSRS. In this study, Cronbach's alpha $=0.72$, indicating that our LGSRS had fair internal consistency and reliability.

2.5. Investigation of Mental State. The Hamilton anxiety scale (HAMA) [24] was used to assess the mental state of the patients, which consisted of two subscales: psychic anxiety and somatic anxiety. The HAMA contains 14 items, and each item is rated on a 5-point Likert scale ranging from grade 0 to grade 4 , which refers to no symptom and extremely severe symptoms, respectively. A global HAMA score of $>6$ indicates 'anxiety.'

In addition, the Hamilton depression scale (HAMD) contains 17 items that were grouped into five structural factors: "anxiety/somatization," "mental disorders," "retardation symptoms," "sleep disturbances," and "weight loss." HAMD uses a 5-point Likert scale ranging from grade 0 to grade 4, referring to no symptoms and extremely severe symptoms, respectively. A global HAMA score of $>7$ indicates 'depression' [25].

Three specially trained professionals administered HAMA and HAMD through conversation and observation.

2.6. Statistical Analysis. The EpiData 3.1 software (The Epidata Association, Odense, Denmark) was used to input data, while the SPSS21.0 software (SPSS Inc., Chicago, IL) was used for statistical analysis. The normal distribution data is expressed as means \pm SEM, while the data with nonnormal distribution were represented by the median and range. Continuous variables were compared parametrically using Student's $t$-test or nonparametrically using the Mann-Whitney $U$ test. Moreover, prevalence was calculated using mean \pm 2 SD of healthy controls as the upper limit of normal, while Spearman's correlation was used to correlate the different scores. Cronbach's alpha was calculated to measure the internal consistency reliability and validity of LGSRS. The difference was statistically significant with $P<0.05$.

\section{Result}

3.1. Demographics Data. Among the 115 FC patients, the average age was $37.87 \pm 8.11$ years old, which included 60 males and 55 females; among the 126 IBS patients, the average age was $36.52 \pm 8.83$ years old, which include 54 males and 72 females; among the 96 healthy controls, the average age was $35.63 \pm 8.37$ years old, which include 45 males and 51 females. According to Rome IV criteria [17, 19], there were 88 IBS-diarrhea patients, 35 IBS-constipation patients, and 3 IBS-mixed patients. No significant differences in age, sex, and BMI were detected between the three groups, see Table 1.

3.2. Different Scores in the 3 Groups. The taste score in the 3 groups (healthy controls, FC, and IBS) was $0.88 \pm 0.81$, $3.13 \pm 1.46$, and $3.57 \pm 1.57$, respectively. The smell score in the 3 groups (healthy controls, FC, and IBS) was $0.63 \pm$ $0.50,2.09 \pm 1.24$, and $2.00 \pm 0.95$, respectively. This revealed a significant difference in taste and smell scores between patients with FC/IBS and the control group. The HAMA score in the 3 groups (healthy controls, FC, and IBS) were $3.19 \pm 1.76,9.56 \pm 5.10$, and $9.19 \pm 4.23$, respectively. The HAMD score in the 3 groups (healthy controls, FC, and IBS) was $3.19 \pm 1.80,10.04 \pm 4.38$, and $9.71 \pm 4.93$, respectively. There was a significant difference in the HAMA score and HAMD score between patients with FC/IBS and the control group. The LGSRS score in the 3 groups (healthy controls, FC, and IBS) was $2.31 \pm 1.40,15.52 \pm 3.10$, and 
TABLE 1: Demographics and characteristics of the three groups (healthy controls, functional constipation, and irritable bowel syndrome) in this study.

\begin{tabular}{|c|c|c|c|c|c|c|}
\hline & Overall $(N=337)$ & Healthy controls $(n=96)$ & $\mathrm{FC}(n=115)$ & IBS $(n=126)$ & $\chi^{2} / t$ & $P$ \\
\hline \multicolumn{7}{|l|}{ Gender } \\
\hline Male $(n)$ & 159 & 45 & 60 & 54 & \multirow{2}{*}{2.099} & \multirow{2}{*}{0.350} \\
\hline Female $(n)$ & 178 & 51 & 55 & 72 & & \\
\hline Age (years, mean $\pm \mathrm{SE}$ ) & $45.71 \pm 10.64$ & $35.63 \pm 8.37$ & $37.87 \pm 8.11$ & $36.52 \pm 8.83$ & 1.744 & 0.087 \\
\hline $\mathrm{BMI}\left(\mathrm{kg} / \mathrm{m}^{2}\right.$, mean $\left.\pm \mathrm{SE}\right)$ & $21.31 \pm 4.03$ & $21.53 \pm 3.67$ & $20.45 \pm 3.05$ & $21.68 \pm 4.83$ & 0.789 & 0.434 \\
\hline
\end{tabular}

FC: functional constipation; IBS: irritable bowel syndrome; body mass index $(\mathrm{BMI})=$ weight $(\mathrm{in} \mathrm{kg}) / \mathrm{height}^{2}\left(\right.$ in $^{2}{ }^{2}$. No statistically significant difference was noted in age, gender, and BMI.

$14.52 \pm 3.57$, respectively. Thus, we found a significant difference in LGSRS scores between patients with FC/IBS and the control group (Table 2).

3.3. Correlation in Different Scores. The obtained results indicated that LGSRS was significantly correlated with the taste score (Spearman's rho $=0.832, P<0.001$ ) in the overall patients' group. LGSRS was significantly correlated with HAMA (Spearman's rho $=0.357, P=0.017$ ) and HAMD (Spearman's rho $=0.377, P=0.012$ ). In addition, the taste score was significantly correlated with HAMD (Spearman's rho $=0.479, P=0.001$, while the smell score was also significantly correlated with HAMD (Spearman's rho $=0.325$, $P=0.031)$. All the above results are represented in Table 3 .

3.4. Taste Survey of the TSS. In the taste survey, 35 and 42 patients of the FC and IBS group found their tastes were different from before, respectively. Interestingly, patients often found that they had a weaker taste of saltiness, sweetness, and sourness; in contrast, the taste of bitterness was stronger. 10 patients with FC reported severe to intolerable change in their sense of taste, while 6 patients with IBS found severe to intolerable change in their gustatory senses (Table 4).

3.5. Smell Survey of the TSS. In the smell survey, 50 patients with FC found their foods smelled different from before, while 48 IBS patients found that their foods smelled different compared to the past. Interestingly, patients often felt their sense of smell diminished. 10 patients with FC found severe to intolerable changes in their sense of smell, while 18 patients with IBS found severe to intolerable alterations in their olfactory senses (Table 5).

3.6. Prevalence of Taste/Smell Abnormalities. Prevalence was calculated using mean $\pm 2 \mathrm{SD}$ of healthy controls as the upper limit of normal. Taste scores (out of 10 points) of healthy controls were $0.88 \pm 0.81$, and thus, the upper limit of normal was 2.50. Similarly, the upper limit of the smell score was 1.63. Therefore, there were $6(6.25 \%), 70$ $(60.87 \%)$, and $90(71.43 \%)$ participants who had taste abnormality, while $0(0.00 \%), 75$ (65.22\%), and $90(71.43 \%)$ participants had smell abnormality in the control group, FC group, and IBS group, respectively. Notably, there were more patients with taste abnormality in the FC $\left(\chi^{2}=67.733, \quad P<0.001\right)$ and IBS $\left(\chi^{2}=94.311, P<0.001\right)$ groups compared to healthy controls. Similarly, there were more patients with smell abnormality in $\mathrm{FC}\left(\chi^{2}=97.136\right.$, $P<0.001)$ and IBS $\left(\chi^{2}=115.325, P<0.001\right)$ than healthy controls.

3.7. Prevalence of Anxiety and Depression. Generally, a global HAMA score of $>6$ indicates 'anxiety,' while a global HAMD score of $>7$ indicates 'depression.' There were $6(6.25 \%), 55$ $(47.83 \%)$, and $60(47.62 \%)$ patients who suffered from anxiety, while $0(0.00 \%), 50(43.48 \%)$, and $72(57.14 \%)$ patients reached the depression criteria in the healthy control group, FC group, and IBS group, respectively. Notably, the number of patients with anxiety in the FC $\left(\chi^{2}=44.007, P<0.001\right)$ and IBS $\left(\chi^{2}=44.635, P<0.001\right)$ groups was more than in healthy controls. Similarly, there were more patients with depression in the FC $\left(\chi^{2}=54.702, P<0.001\right)$ and IBS $\left(\chi^{2}=81.189, P<0.001\right)$ groups than in healthy patients.

3.8. Comparative Analysis of Different Scores between Male and Female. The taste, smell, HAMD, and LGSRS scores in the IBS female group were $4.08 \pm 1.26,2.17 \pm 1.15,11.25 \pm$ 5.36 , and $15.17 \pm 4.02$, respectively; while the taste, smell, HAMD, and LGSRS scores in the IBS male group were $2.89 \pm 1.61, \quad 1.78 \pm 0.42, \quad 7.67 \pm 3.01, \quad$ and $12.44 \pm 3.98$, respectively. There were significant differences in the taste, smell, HAMD, and LGSRS scores between the female and male IBS groups $(P<0.050)$. However, the taste, smell, HAMA, HAMD, and LGSRS scores did not differ in female FC patients compared to male patients (Table 6).

\section{Discussion}

The chemical senses of taste and smell are essential to life. Physiological TSCs alert us to dangers (e.g., gas leaks), prevent us from ingesting toxins, and support our oral nutrition [26]. However, pathologically TSCs might contribute to an increased risk of malnutrition (under- or overnutrition) [27], low mood, diminished social interaction, and reduced quality of life [28]. This study shows that taste and smell abnormalities in patients with FC and IBS were not uncommon. Importantly, the taste abnormalities were positively correlated with the severity of lower gastrointestinal symptoms. A breakdown of individual taste complaints showed that most patients noted their gustatory functions were 
TABLE 2: Taste, smell, HAMA, and HAMD scores and lower gastrointestinal symptoms rating score in the 3 study groups (healthy controls, functional constipation, and irritable bowel syndrome).

\begin{tabular}{lccc}
\hline & Healthy controls $(n=96)$ & FC $(n=115)$ & IBS $(n=126)$ \\
\hline Taste score (out of 10 points) (mean \pm SD) & $0.88 \pm 0.81$ & $3.13 \pm 1.46$ & $3.57 \pm 1.57$ \\
$P$ value vs. healthy controls & & $t=5.61, P<0.001$ & $t=6.24, P<0.001$ \\
Smell score(out of 6 points) (mean \pm SD) & $0.63 \pm 0.50$ & $2.09 \pm 1.24$ & $2.00 \pm 0.95$ \\
$P$ value vs. healthy controls & & $t=5.26, P<0.001$ & $t=4.46, P<0.001$ \\
HAMA(out of 64 points) (mean \pm SD) & $3.19 \pm 1.76$ & $9.56 \pm 5.10$ & $t=4.79, P<0.001$ \\
$P$ value vs. healthy controls & & $10.04 \pm 4.38$ & $t=5.33, P<0.001$ \\
HAMD (out of 72 points) (mean \pm SD) & $3.19 \pm 1.80$ & $t=5.90, P<0.001$ & $9.71 \pm 4.93$ \\
$P$ value vs. healthy controls & & $15.52 \pm 3.10$ & $t=5.03, P<0.001$ \\
LGSRS (out of 30 points) (mean \pm SD) & $2.31 \pm 1.40$ & $t=15.89, P<0.001$ & $t=12.90, P<0.001$ \\
$P$ value vs. healthy controls & &
\end{tabular}

FC: functional constipation; IBS: irritable bowel syndrome; LGSRS: lower gastrointestinal symptoms rating scale.

TABLE 3: Correlation among taste, smell, HAMA, HAMD, and LGSRS scores for functional constipation and irritable bowel syndrome patients.

\begin{tabular}{|c|c|c|c|c|c|c|}
\hline & \multicolumn{2}{|c|}{$\mathrm{FC}(n=115)$} & \multicolumn{2}{|c|}{ IBS $(n=126)$} & \multicolumn{2}{|c|}{ Overall patients $(n=241)$} \\
\hline & Spearman's rho & $P$ & Spearman's rho & $P$ & Spearman's rho & $P$ \\
\hline Taste vs. LGSRS & 0.796 & $<0.001$ & 0.890 & $<0.001$ & 0.832 & $<0.001$ \\
\hline Smell vs. LGSRS & 0.046 & 0.834 & 0.702 & $<0.001$ & 0.271 & 0.075 \\
\hline HAMA vs. LGSRS & 0.010 & 0.964 & 0.627 & 0.002 & 0.357 & 0.017 \\
\hline HAMD vs. LGSRS & 0.103 & 0.641 & 0.585 & 0.005 & 0.377 & 0.012 \\
\hline Taste vs. smell & 0.088 & 0.690 & 0.672 & 0.001 & 0.319 & 0.035 \\
\hline Taste vs. HAMA & 0.177 & 0.419 & 0.673 & 0.001 & 0.260 & 0.088 \\
\hline Taste vs. HAMD & 0.232 & 0.288 & 0.690 & 0.001 & 0.479 & 0.001 \\
\hline Smell vs. HAMA & 0.790 & $<0.001$ & 0.605 & 0.004 & 0.185 & 0.230 \\
\hline Smell vs. HAMD & 0.807 & $<0.001$ & 0.339 & 0.132 & 0.325 & 0.031 \\
\hline
\end{tabular}

FC: functional constipation; IBS: irritable bowel syndrome; LGSRS: lower gastrointestinal symptoms rating scale. Data are expressed as Spearman's correlation coefficient rho with $P$ values.

weaker when eating salty, sweet, and sour food items but were stronger when tasting bitter food, consistent with previous results reported by Silke et al. [4]. Additionally, patients often suffer from mental disorders of anxiety and depression, and higher HAMA score/HAMD scores were positively correlated with the severity of lower gastrointestinal symptoms. Notably, the HAMD score was positively correlated with the smell and taste scores of all subjects in the study. Interestingly, we compared the difference of evaluated characteristics in the current study and found that female IBS patients reported more severe alterations in taste and smell, lower gastrointestinal symptoms, and depression when compared with male patients, which is similar to a previous study [29]. However, taste and smell changes did not differ in female FC patients compared to male patients.

Presently, the mechanism of the changes in taste and smell caused by FC and IBS symptoms has not yet been fully elucidated, but these may be related to abnormal brain-gut axis interactions [30-32]. The brain-gut axis is recognized as a complex bidirectional communication system between the gastrointestinal tract and the brain, with many cellular and molecular pathways acting along this axis [33]. Kidd et al. [34] confirmed that enterochromaffin cells might be a luminal sensor for odorants and taste molecules followed by regulating gut motility. Steinbach et al. [35] demonstrated that scores of odor threshold were decreased while the scores of odor identification and odor discrimination were increased in IBS patients compared to the control, supporting the idea of a central etiology of IBS. Furthermore, a previous review concluded that altered gut microbiota in IBS produced a complex interaction with genetic variants dispersed in the human genome (intrinsic factors) and led to individual epigenetic prints, including many of the genes and molecular mechanisms involved in taste biology [36, 37]. Meanwhile, studies have also found that the morphology and metabolism of the insula in depressive patients were significantly altered. The insular lobe is a cortical structure located deep in the brain tissue and participates in processing taste and smell $[38,39]$. Aschenbrenner et al. [40] found that anorexic patients had a significant decrease in taste and olfactory function, which was associated with higher depression scores. This study is the first to demonstrate that a large 
TABLE 4: Breakdown of the detailed taste complaints from the taste and smell survey.

\begin{tabular}{|c|c|c|c|c|}
\hline & & Healthy controls $(n=96)$ & $\mathrm{FC}(n=115)$ & IBS $(n=126)$ \\
\hline \multicolumn{5}{|c|}{ Foods taste different than they used to } \\
\hline Yes & & $12(12.50)$ & $35(30.43)$ & $42(33.33)$ \\
\hline No & & $84(87.50)$ & $80(69.57)$ & $84(66.67)$ \\
\hline \multicolumn{5}{|c|}{$\begin{array}{l}\text { I have noticed a change in my sense of } \\
\text { taste }\end{array}$} \\
\hline Yes & & $6(6.25)$ & $30(26.09)$ & $36(28.57)$ \\
\hline No & & $90(93.75)$ & $85(73.91)$ & $90(71.43)$ \\
\hline \multicolumn{5}{|c|}{ I have a persistent bad taste in mouth } \\
\hline Yes & & $6(6.25)$ & $45(39.13)$ & $60(47.62)$ \\
\hline No & & $90(93.75)$ & $70(60.87)$ & $66(52.38)$ \\
\hline \multicolumn{5}{|c|}{ Drugs interfere with my sense of taste } \\
\hline Yes & & $0(0.00)$ & $40(34.78)$ & $48(38.10)$ \\
\hline No & & $96(100.00)$ & $75(65.22)$ & $78(61.90)$ \\
\hline \multicolumn{5}{|c|}{$\begin{array}{l}\text { Comparison of sense of taste now to } \\
\text { before diagnosis }\end{array}$} \\
\hline \multicolumn{5}{|c|}{ I have noticed a change in salt } \\
\hline & Salt tastes stronger & $6(6.25)$ & $10(8.70)$ & $18(14.29)$ \\
\hline \multirow[t]{2}{*}{ Yes } & Salt tastes weaker & $6(6.25)$ & $40(34.78)$ & $36(28.57)$ \\
\hline & Cannot taste & $0(0.00)$ & $5(4.35)$ & $6(4.76)$ \\
\hline No & & $84(87.50)$ & $60(52.17)$ & $66(52.38)$ \\
\hline \multicolumn{5}{|c|}{ I have noticed a change in sweet } \\
\hline & Sweet tastes stronger & $12(12.50)$ & $10(8.70)$ & $18(14.29)$ \\
\hline \multirow[t]{2}{*}{ Yes } & Sweet tastes weaker & $6(6.25)$ & $20(17.39)$ & $36(28.57)$ \\
\hline & Cannot taste & $0(0.00)$ & $0(0.00)$ & $0(0.00)$ \\
\hline No & & $78(81.25)$ & $85(73.91)$ & $72(57.14)$ \\
\hline \multicolumn{5}{|c|}{ I have noticed a change in sour } \\
\hline & Sour tastes stronger & $0(0.00)$ & $15(13.04)$ & $12(9.53)$ \\
\hline \multirow[t]{2}{*}{ Yes } & Sour tastes weaker & $12(12.50)$ & $20(17.39)$ & $30(23.81)$ \\
\hline & Cannot taste & $0(0.00)$ & $5(4.35)$ & $6(4.76)$ \\
\hline No & & $84(87.50)$ & $75(65.22)$ & $78(61.90)$ \\
\hline \multicolumn{5}{|c|}{ I have noticed a change in bitter } \\
\hline & Bitter tastes stronger & $6(6.25)$ & $20(17.39)$ & $36(28.57)$ \\
\hline \multirow[t]{2}{*}{ Yes } & Bitter tastes weaker & $6(6.25)$ & $15(13.04)$ & $24(19.05)$ \\
\hline & Cannot taste & $0(0.00)$ & $5(4.35)$ & $6(4.76)$ \\
\hline No & & $84(87.50)$ & $75(65.22)$ & $60(47.62)$ \\
\hline \multicolumn{5}{|c|}{ Rate abnormal sense of taste } \\
\hline Insig & & $90(93.75)$ & $80(69.57)$ & $96(76.19)$ \\
\hline Mild & & $6(6.25)$ & $25(21.74)$ & $24(19.05)$ \\
\hline Sever & & $0(0.00)$ & $10(8.70)$ & $6(4.76)$ \\
\hline
\end{tabular}

Data are expressed as $n$ (\%). FC: functional constipation; IBS: irritable bowel syndrome.

number of patients with FC and IBS are afflicted by TSCs as well as anxiety and depression, which is in line with previous studies $[41,42]$. In addition, we detected a positive association between depression score and TSCs as well as the severity of lower gastrointestinal symptoms.

So far, there is a lack of definitive biomedical solutions to alleviate or restore taste and smell functions and adjust individuals' TSCs [42]. Studies have reported how patients find ways to avoid unpleasant taste or smell experiences or man- age the social impact of TSCs [43]. Based on patients' understanding of the TSCs, proactive patients with a positive attitude will take the initiative to manage their eating habits, such as eating healthier (less fat, more vegetables), choosing seasonings to adjust the taste of food, avoid odor, to name a few [44]. Therefore, FC and IBS patients need to be aware of the potential changes in their taste and smell to be able to manage their eating habits. Meanwhile, physicians should also pay more attention to this phenomenon 
TABLE 5: Breakdown of the detailed smell complaints from the taste and smell survey.

\begin{tabular}{|c|c|c|c|}
\hline & Healthy controls $(n=96)$ & $\mathrm{FC}(n=115)$ & IBS $(n=126)$ \\
\hline \multicolumn{4}{|c|}{ I have noticed a change in my sense of smell } \\
\hline Yes & $12(12.50)$ & $50(43.48)$ & $48(38.10)$ \\
\hline No & $84(87.50)$ & $65(56.52)$ & $78(61.90)$ \\
\hline \multicolumn{4}{|c|}{ Foods smell different than they used to } \\
\hline Yes & $12(12.50)$ & $55(47.83)$ & $54(42.86)$ \\
\hline No & $84(87.50)$ & $60(52.17)$ & $72(57.14)$ \\
\hline \multicolumn{4}{|c|}{ Specific drugs interfere with my sense of smell } \\
\hline Yes & $6(6.25)$ & $30(26.09)$ & $36(28.57)$ \\
\hline No & $90(93.75)$ & $85(73.91)$ & $90(71.43)$ \\
\hline \multicolumn{4}{|c|}{ I have noticed a change in odors } \\
\hline Stronger & $12(12.50)$ & $25(21.74)$ & $18(14.28)$ \\
\hline Weaker & $6(6.25)$ & $40(34.78)$ & $24(19.05)$ \\
\hline No change & $78(81.25)$ & $50(43.48)$ & $84(66.67)$ \\
\hline \multicolumn{4}{|c|}{ Rate abnormal sense of smell } \\
\hline Insignificant & $84(87.50)$ & $85(73.91)$ & $72(57.14)$ \\
\hline Mild to moderate & $12(12.50)$ & $20(17.39)$ & $36(28.57)$ \\
\hline Severe to intolerable & $0(0.00)$ & $10(8.70)$ & $18(14.29)$ \\
\hline
\end{tabular}

Data are expressed as $n$ (\%). FC: functional constipation; IBS: irritable bowel syndrome.

TABLE 6: Gender-related differences of taste, smell, HAMA, HAMD, and LGSRS scores in FC and IBS patients.

\begin{tabular}{lcccccccc}
\hline & & FC $(N=115)$ & & & \multicolumn{3}{c}{ IBS $(N=126)$} \\
& Female $(n=55)$ & Male $(n=60)$ & $t$ & $P$ & Female $(n=72)$ & Male $(n=54)$ & $t$ \\
\hline Taste score & $3.32 \pm 1.49$ & $3.07 \pm 140$ & 0.55 & 0.583 & $4.08 \pm 1.26$ & $2.89 \pm 1.61$ & 4.67 & $<0.001$ \\
Smell score & $1.89 \pm 0.86$ & $2.21 \pm 0.73$ & 1.41 & 0.163 & $2.17 \pm 1.15$ & $1.78 \pm 0.42$ & 2.37 & 0.020 \\
HAMA & $9.90 \pm 3.13$ & $9.18 \pm 3.28$ & 1.19 & 0.210 & $9.42 \pm 4.55$ & $8.86 \pm 2.82$ & 1.07 & 0.168 \\
HAMD & $10.36 \pm 3.11$ & $9.56 \pm 3.47$ & 0.97 & 0.332 & $11.25 \pm 5.36$ & $7.67 \pm 3.01$ & 4.41 & $<0.001$ \\
LGSRS & $14.21 \pm 4.02$ & $13.89 \pm 3.14$ & 0.54 & 0.589 & $15.17 \pm 4.02$ & $12.44 \pm 3.98$ & 3.77 & $<0.001$ \\
\hline
\end{tabular}

FC: functional constipation; IBS: irritable bowel syndrome; LGSRS: lower gastrointestinal symptoms rating scale.

because our study shows that this may help better predict and understand the severity of lower gastrointestinal symptoms and TSC's association with psychiatric comorbidity and symptoms.

\section{Limitations}

There following are some shortcomings in this study: (1) The study used food-related smell and taste scores rather than objective tests $[45,46]$, giving rise to inherent recall bias that may result in participants underscoring or exaggerating their senses and feelings unconsciously. (2) Our sample size was quite small; therefore, larger-scale and multicenter studies are warranted in the future. Consequently, the difficulty of helping patients with taste and smell changes underscores the need of a diagnostic and evaluation tool for taste and smell changes and evidence-based treatment strategies. Meanwhile, the relationship among TSCSs, LGSRS, and psychological comorbidities is still unclear and needs further research clinically.

\section{Conclusions}

Taste and smell changes are common but are often the neglected symptoms in patients with digestive disorders. In this study, we found that changes in taste and smell were common in FC and IBS patients. The change in patients' taste is related to their LGSRS, and these patients were more likely to suffer from anxiety and depression, which were also found to be significantly correlated with LGSRS. Awareness of this high prevalence of taste/smell abnormalities and the consequent psychological alterations among patients with FC and IBS may also help better understand the severity of their lower gastrointestinal symptoms. Therefore, more high-quality evidence is warranted to guide future clinical practice.

\section{Data Availability}

All data generated or analyzed during this study are included in this published article. The data used to support the 
findings of this study are available from the corresponding author upon request. Data were collected by authorized researchers.

\section{Ethical Approval}

The protocol was approved by Ethics Committee of Anhui Provincial Hospital. The guidelines of Helsinki Declaration were followed.

\section{Consent}

All patients joined the study with written informed consent for research use of their survey data.

\section{Disclosure}

The abstract of this study is accepted for E-poster in the 4th international meeting on intestinal diseases.

\section{Conflicts of Interest}

The authors declare that they have no conflicts of interests.

\section{Authors' Contributions}

Yue Yu contributed to the design the study. Jie Liu, Chaolan Lv, Dandan Wu, Ying Wang, and Yue Yu conducted the study and collected data. Jie Liu, Chaolan Lv, Chenyu Sun, and Ce Cheng interpreted the data. Jie Liu, Chaolan Lv, Chenyu Sun, and Ce Cheng made the draft of the manuscript. Yue Yu contributed to the revision of the study and provided critical opinion. All authors read and approved the final manuscript. Jie Liu, Chaolan Lv, and Dandan Wu contributed equally to this study.

\section{Acknowledgments}

The study was supported by the External Science and Technology Cooperation Planning Projects of Anhui Province of China (No. 1604b0602021).

\section{References}

[1] P. Pearodwong, R. Muns, and P. Tummaruk, "Prevalence of constipation and its influence on post-parturient disorders in tropical sows," Tropical Animal Health and Production, vol. 48, no. 3, pp. 525-531, 2016.

[2] Y. Long, Z. Huang, Y. Deng et al., "Prevalence and risk factors for functional bowel disorders in South China: a population based study using the Rome III criteria," Neurogastroenterology \& Motility, vol. 29, no. 1, article e12897, p. 10, 2017.

[3] A. Kabadi, M. Saadi, R. Schey, and H. P. Parkman, "Taste and smell disturbances in patients with gastroparesis and gastroesophageal reflux disease," Journal of neurogastroenterology and motility, vol. 23, no. 3, pp. 370-377, 2017.

[4] S. Steinbach, W. Reindl, A. Dempfle et al., "Smell and taste in inflammatory bowel disease," PLoS One, vol. 8, no. 9, article e73454, 2013.

[5] R. L. Doty, "Age-related deficits in taste and smell," Otolaryngologic Clinics of North America, vol. 51, no. 4, pp. 815-825, 2018.
[6] B. M. Bernhardson, C. Tishelman, and L. E. Rutqvist, "Selfreported taste and smell changes during cancer chemotherapy," Supportive Care in Cancer, vol. 16, no. 3, pp. 275-283, 2008.

[7] I. IJpma, R. J. Renken, J. A. Gietema et al., "Changes in taste and smell function, dietary intake, food preference, and body composition in testicular cancer patients treated with cisplatin-based chemotherapy," Clinical Nutrition, vol. 36, no. 6, pp. 1642-1648, 2017.

[8] K. Sánchez-Lara, R. Sosa-Sánchez, D. Green-Renner et al., "Influence of taste disorders on dietary behaviors in cancer patients under chemotherapy," Nutrition Journal, vol. 9, no. 1, p. 15, 2010.

[9] Y. C. de Vries, E. Helmich, M. D. Karsten, S. Boesveldt, R. M. Winkels, and H. van Laarhoven, "The impact of chemosensory and food-related changes in patients with advanced oesophagogastric cancer treated with capecitabine and oxaliplatin: a qualitative study," Supportive Care in Cancer, vol. 24, no. 7, pp. 3119-3126, 2016.

[10] E. Kinnaird, C. Stewart, and K. Tchanturia, "The relationship of autistic traits to taste and olfactory processing in anorexia nervosa," Molecular Autism, vol. 11, no. 1, p. 25, 2020.

[11] S. Boesveldt, J. R. Yee, M. K. McClintock, and J. N. Lundström, "Olfactory function and the social lives of older adults: a matter of sex," Scientific Reports, vol. 7, no. 1, article 45118, 2017.

[12] H. Taalman, C. Wallace, and R. Milev, "Olfactory functioning and depression: a systematic review," Frontiers in Psychiatry, vol. 8, p. 190, 2017.

[13] P. Kohli, Z. M. Soler, S. A. Nguyen, J. S. Muus, and R. J. Schlosser, "The association between olfaction and depression: a systematic review," Chemical Senses, vol. 41, no. 6, pp. 479-486, 2016.

[14] K. Hur, J. S. Choi, M. Zheng, J. Shen, and B. Wrobel, “Association of alterations in smell and taste with depression in older adults," Laryngoscope investigative otolaryngology, vol. 3, no. 2, pp. 94-99, 2018.

[15] M. R. Khan, M. Saha, M. A. Mamun et al., "Upper GIT endoscopic evaluation and psychological state assessment of patients with globus sensation," Mymensingh Medical Journal, vol. 28, no. 2, pp. 405-409, 2019.

[16] A. E. Heald, C. F. Pieper, and S. S. Schiffman, "Taste and smell complaints in HIV-infected patients," AIDS, vol. 12, no. 13, pp. 1667-1674, 1998.

[17] D. A. Drossman, "Functional Gastrointestinal Disorders: History, Pathophysiology, Clinical Features, and Rome IV," Gastroenterology, vol. 150, no. 6, pp. 1262-1279.e2, 2016.

[18] S. S. Rao, K. Rattanakovit, and T. Patcharatrakul, "Diagnosis and management of chronic constipation in adults," Nature Reviews. Gastroenterology \& Hepatology, vol. 13, no. 5, pp. 295-305, 2016.

[19] B. E. Lacy and N. K. Patel, "Rome criteria and a diagnostic approach to irritable bowel syndrome," Journal of Clinical Medicine, vol. 6, no. 11, p. 99, 2017.

[20] B. M. Bernhardson, C. Tishelman, and L. E. Rutqvist, "Taste and smell changes in patients receiving cancer chemotherapy: distress, impact on daily life, and self-care strategies," Cancer Nursing, vol. 32, no. 1, pp. 45-54, 2009.

[21] K. Belqaid, Y. Orrevall, J. McGreevy et al., "Self-reported taste and smell alterations in patients under investigation for lung cancer," Acta Oncologica, vol. 53, no. 10, pp. 1405-1412, 2014.

[22] K. Belqaid, C. Tishelman, J. McGreevy et al., "A longitudinal study of changing characteristics of self-reported taste and 
smell alterations in patients treated for lung cancer," European Journal of Oncology Nursing, vol. 21, pp. 232-241, 2016.

[23] K. R. Kulich, A. Madisch, F. Pacini et al., "Reliability and validity of the Gastrointestinal Symptom Rating Scale (GSRS) and Quality of Life in Reflux and Dyspepsia (QOLRAD) questionnaire in dyspepsia: a six-country study," Health and Quality of Life Outcomes, vol. 6, no. 1, p. 12, 2008.

[24] Z. J. Xie, N. Han, S. Law et al., "The efficacy of group cognitivebehavioural therapy plus duloxetine for generalised anxiety disorder versus duloxetine alone," Acta neuropsychiatrica, vol. 31, no. 6, pp. 316-324, 2019.

[25] J. Lu, L. Shi, D. Huang et al., "Depression and structural factors are associated with symptoms in patients of irritable bowel syndrome with diarrhea," Journal of Neurogastroenterology and Motility, vol. 26, no. 4, pp. 505-513, 2020.

[26] T. D. Brisbois, J. L. Hutton, V. E. Baracos, and W. V. Wismer, "Taste and smell abnormalities as an independent cause of failure of food intake in patients with advanced cancer-an argument for the application of sensory science," Journal of Palliative Care, vol. 22, no. 2, pp. 111-114, 2006.

[27] L. McLaughlin and S. Mahon, "Understanding taste dysfunction in patients with cancer," Clinical Journal of Oncology Nursing, vol. 16, no. 2, pp. 171-178, 2012.

[28] J. H. Hong, P. Omur-Ozbek, B. T. Stanek et al., "Taste and odor abnormalities in cancer patients," The Journal of Supportive Oncology, vol. 7, no. 2, pp. 58-65, 2009.

[29] O. Y. Lee, E. A. Mayer, M. Schmulson, L. Chang, and B. Naliboff, "Gender-related differences in IBS symptoms," The American Journal of Gastroenterology, vol. 96, no. 7, pp. 2184-2193, 2001.

[30] D. A. Drossman and W. L. Hasler, "Rome IV-Functional GI Disorders: Disorders of Gut-Brain Interaction," Gastroenterology, vol. 150, no. 6, pp. 1257-1261, 2016.

[31] A. Ancona, C. Petito, I. Iavarone et al., "The gut-brain axis in irritable bowel syndrome and inflammatory bowel disease," Digestive and Liver Disease, vol. 53, no. 3, pp. 298305, 2021.

[32] S. Y. So and T. C. Savidge, "Sex-bias in irritable bowel syndrome: linking steroids to the gut-brain axis," Frontiers in Endocrinology, vol. 12, article 684096, 2021.

[33] A. Baj, E. Moro, M. Bistoletti, V. Orlandi, F. Crema, and C. Giaroni, "Glutamatergic signaling along the microbiotagut-brain axis," International Journal of Molecular Sciences, vol. 20, no. 6, p. 1482, 2019.

[34] M. Kidd, I. M. Modlin, B. I. Gustafsson, I. Drozdov, O. Hauso, and R. Pfragner, "Luminal regulation of normal and neoplastic human EC cell serotonin release is mediated by bile salts, amines, tastants, and olfactants," American Journal of Physiology. Gastrointestinal and Liver Physiology, vol. 295, no. 2, pp. G260-G272, 2008.

[35] S. Steinbach, W. Reindl, C. Kessel et al., "Olfactory and gustatory function in irritable bowel syndrome," European Archives of Oto-Rhino-Laryngology, vol. 267, no. 7, pp. 1081-1087, 2010.

[36] M. Gazouli, M. M. Wouters, L. Kapur-Pojskić et al., "Lessons learned - resolving the enigma of genetic factors in IBS," Nature Reviews. Gastroenterology \& Hepatology, vol. 13, no. 2, pp. 77-87, 2016.

[37] S. A. Gravina, G. L. Yep, and M. Khan, "Human biology of taste," Annals of Saudi Medicine, vol. 33, no. 3, pp. 217-222, 2013.
[38] L. Su, Y. Cai, Y. Xu, A. Dutt, S. Shi, and E. Bramon, "Cerebral metabolism in major depressive disorder: a voxel-based metaanalysis of positron emission tomography studies," BMC Psychiatry, vol. 14, no. 1, p. 321, 2014.

[39] R. Lanzenberger, P. Baldinger, A. Hahn et al., "Global decrease of serotonin-1A receptor binding after electroconvulsive therapy in major depression measured by PET," Molecular Psychiatry, vol. 18, no. 1, pp. 93-100, 2013.

[40] K. Aschenbrenner, N. Scholze, P. Joraschky, and T. Hummel, "Gustatory and olfactory sensitivity in patients with anorexia and bulimia in the course of treatment," Journal of Psychiatric Research, vol. 43, no. 2, pp. 129-137, 2008.

[41] C. Lee, E. Doo, J. M. Choi et al., "The increased level of depression and anxiety in irritable bowel syndrome patients compared with healthy controls: systematic review and metaanalysis," Journal of Neurogastroenterology and Motility, vol. 23, no. 3, pp. 349-362, 2017.

[42] X. Li, R. Feng, H. Wu et al., "Psychological characteristics and GoNogo research of patients with functional constipation," Medicine (Baltimore), vol. 95, no. 52, article e5685, 2016.

[43] O. Sevryugin, P. Kasvis, M. Vigano, and A. Vigano, "Taste and smell disturbances in cancer patients: a scoping review of available treatments," Supportive Care in Cancer, vol. 29, no. 1, pp. 49-66, 2021.

[44] K. Belqaid, C. Tishelman, Y. Orrevall, E. Månsson-Brahme, and B. M. Bernhardson, "Dealing with taste and smell alterations-a qualitative interview study of people treated for lung cancer," PLoS One, vol. 13, no. 1, article e0191117, 2018.

[45] L. E. Spotten, C. A. Corish, C. M. Lorton et al., "Subjective and objective taste and smell changes in cancer," Annals of Oncology, vol. 28, no. 5, pp. 969-984, 2017.

[46] B. N. Landis, A. Welge-Luessen, A. Brämerson et al., "'Taste strips" - a rapid, lateralized, gustatory bedside identification test based on impregnated filter papers," Journal of Neurology, vol. 256, no. 2, pp. 242-248, 2009. 